\title{
Modest Impact on Risk for Autism Spectrum Disorder of Rare Copy Number Variants at 15q11.2, Specifically Breakpoints 1 to 2
}

\author{
Pauline Chaste, Stephan J. Sanders, Kommu N. Mohan, Lambertus Klei, Youeun Song, \\ Michael T. Murtha, Vanessa Hus, Jennifer K. Lowe, A. Jeremy Willsey, Daniel Moreno-De-Luca, \\ Timothy W. Yu, Eric Fombonne, Daniel Geschwind, Dorothy E. Grice, David H. Ledbetter, \\ Catherine Lord, Shrikant M. Mane, Donna M. Martin, Eric M. Morrow, Christopher A. Walsh, \\ James S. Sutcliffe, Matthew W. State, Christa Lese Martin, Bernie Devlin, Arthur L. Beaudet, \\ Edwin H. Cook Jr., and Soo-Jeong Kim
}

\begin{abstract}
The proximal region of chromosome 15 is one of the genomic hotspots for copy number variants (CNVs). Among the rearrangements observed in this region, CNVs from the interval between the common breakpoints 1 and 2 (BP1 and BP2) have been reported cosegregating with autism spectrum disorder (ASD). Although evidence supporting an association between BP1-BP2 CNVs and autism accumulates, the magnitude of the effect of BP1-BP2 CNVs remains elusive, posing a great challenge to recurrence-risk counseling. To gain further insight into their pathogenicity for ASD, we estimated the penetrance of the BP1-BP2 CNVs for ASD as well as their effects on ASD-related phenotypes in a well-characterized ASD sample ( $\mathrm{n}=2525$ families). Transmission disequilibrium test revealed significant preferential transmission only for the duplicated chromosome in probands (20T:9NT). The penetrance of the BP1-BP2 CNVs for ASD was low, conferring additional risks of $0.3 \%$ (deletion) and $0.8 \%$ (duplication). Stepwise regression analyses suggest a greater effect of the CNVs on ASD-related phenotype in males and when maternally inherited. Taken together, the results are consistent with BP1-BP2 CNVs as risk factors for autism. However, their effect is modest, more akin to that seen for common variants. To be consistent with the current American College of Medical Genetics guidelines for interpretation of postnatal CNV, the BP1-BP2 deletion and duplication CNVs would probably best be classified as variants of uncertain significance (VOUS): they appear to have an impact on risk, but one so modest that these CNVs do not merit pathogenic status. Autism Res 2014, 7: 355-362. (c) 2014 International Society for Autism Research, Wiley Periodicals, Inc.
\end{abstract}

Keywords: 15q11.2; deletion; duplication; penetrance; autism

\section{Introduction}

While converging results underscore the importance of rare de novo events of large effect on autism spectrum disorder (ASD) [Pinto et al., 2010; Sanders et al., 2011], it has proven challenging to estimate the contribution of inherited copy number variants (CNVs) [Bucan et al., 2009; Girirajan, Johnson et al., 2013; Krumm et al., 2013; Morrow et al., 2008; Pinto et al., 2010; Sanders et al., 2011]. There is no doubt, however, that some rare inherited CNVs substantially increase risk, e.g. deletion or duplication of loci affected by recurrent de novo CNVs,

From the Department of Psychiatry, University of Pittsburgh School of Medicine, Pittsburgh, Pennsylvania (P.C., L.K., Y.S., B.D.); FondaMental Foundation, Créteil, France (P.C.); Department of Genetics, Yale University School of Medicine, New Haven, Connecticut (S.J.S., A.J.W., M.W.S.); Department of Psychiatry, University of California, San Francisco, California (S.J.S., A.J.W., M.W.S.); Department of Biological Sciences, BITS PilaniHyderabad Campus, Hyderabad, India (K.N.M.); Department of Human and Molecular Genetics, Baylor College of Medicine, Houston, Texas (K.N.M., A.L.B.); Program on Neurogenetics, Yale University School of Medicine, New Haven, Connecticut (M.T.M., D.M.); Department of Psychology, University of Michigan, Ann Arbor, Michigan (V.H.); Neurogenetics Program, Department of Neurology and Center for Autism Research and Treatment, Semel Institute, David Geffen School of Medicine, University of California, Los Angeles, California (J.K.L., D.G.); Department of Psychiatry, Yale University School of Medicine, New Haven, Connecticut (D.M.); Division of Genetics, Children's Hospital Boston, Harvard Medical School, Boston, Massachusetts (T.W.Y.); Department of Psychiatry, Oregon Health \& Science University, Portland, Oregon (E.F.); Department of Psychiatry, Icahn School of Medicine at Mount Sinai, New York, New York (D.E.G.); Autism and Developmental Medicine Institute, Geisinger Health System, Danville, Pennsylvania (D.H.L., C.L.M.); Center for Autism and the Developing Brain, Weill Cornell Medical College, White Plains, New York (C.L.); Yale Center for Genome Analysis, Yale University, Orange, Connecticut (S.M.M.); Departments of Pediatrics and Human Genetics, University of Michigan Medical Center, Ann Arbor, Michigan (D.M.M.); Department of Molecular Biology, Cell Biology and Biochemistry, Brown University, Providence, Rhode Island (E.M.M.); Department of Psychiatry and Human Behavior, Brown University, Providence, Rhode Island (E.M.M.); Howard Hughes Medical Institute and Division of Genetics, Children's Hospital Boston, and Neurology and Pediatrics, Center for Life Sciences, Harvard Medical School, Boston, Massachusetts (C.A.W.); Departments of Molecular Physiology \& Biophysics and Psychiatry, Vanderbilt Brain Institute, Vanderbilt University, Nashville, Tennessee, (J.S.S.); Institute for Juvenile Research, Department of Psychiatry, University of Illinois, Chicago, Illinois (E.H.C.); Center for Integrative Brain Research, Seattle Children's Research Institute, Department of Psychiatry and Behavioral Science, University of Washington, Seattle, Washington (S.-J.K.)

Received October 24, 2013; accepted for publication March 19, 2014

Address for correspondence and reprints: Pauline Chaste, Department of Psychiatry, University of Pittsburgh School of Medicine, 3811 O'Hara Street, Pittsburgh, PA 15213. E-mail: chastept@upmc.edu; Soo-Jeong Kim, Center for Integrative Brain Research, Seattle Children’s Research Institute, 1900 9th Avenue, Seattle, WA 98101. E-mail: kimsooj@uw.edu

Published online 12 May 2014 in Wiley Online Library (wileyonlinelibrary.com)

DOI: 10.1002/aur.1378

(c) 2014 International Society for Autism Research, Wiley Periodicals, Inc. 
notably 16p11.2 [Sanders et al., 2011; Weiss et al., 2008]. Still, at the population level, the contribution to risk from individual inherited variants of large effect should be small because the individual variants are subject to natural selection [Devlin \& Scherer, 2012], while rare inherited variants that modestly increase risk could play a substantial role in the genetic architecture of the disorder.

The 15q11.2-q13 region harbors five common breakpoints (BP1, BP2, BP3, BP4, and BP5), each breakpoint corresponding to a complex set of segmental duplications [Christian, Fantes, Mewborn, Huang, \& Ledbetter, 1999]. While the large effect of BP1-BP3, BP2-BP3, and BP4-BP5 $\mathrm{CNVs}$ on neurodevelopment has been well established [Cook et al., 1997; Girirajan, Dennis et al., 2013; Moreno-De-Luca et al., 2012; Shen et al., 2010], the picture is considerably less clear for the effects of BP1-BP2 CNVs. The BP1-BP2 genomic interval within band 15q11.2 (henceforth "BP1-BP2" and "15q11.2" are used synonymously.) encompasses four genes not known to be imprinted: NIPA1, NIPA2, CYFIP1, and TUBGCP5. Interestingly, while the BP2-BP3 region is considered the critical region for Prader-Willi syndrome (PWS) and Angelman syndrome (AS), some have reported a correlation between the type of deletion (i.e., BP1-BP3 deletion vs. BP2-BP3 deletion) and the severity of the phenotype [Butler, Bittel, Kibiryeva, Talebizadeh, \& Thompson, 2004; Hartley, Maclean, Butler, Zarcone, \& Thompson, 2005; Sahoo et al., 2007]. Moreover, data accumulate to support the role of BP1-BP2 deletion, and to a lesser extent duplication, as risk factors for several neurodevelopmental disorders, such as schizophrenia, developmental delay, intellectual disability with or without dysmorphism, epilepsy, and ASD [Abdelmoity et al., 2012; Burnside et al., 2011; de Kovel et al., 2010; Doornbos et al., 2009; Murthy et al., 2007; Sempere Perez et al., 2011; van der Zwaag et al., 2010; von der Lippe, Rustad, Heimdal, \& Rodningen, 2011; Vassos et al., 2010]. The magnitude of their effect, however, remains uncertain.

A primary concern for genetic counseling is how much a BP1-BP2 CNV increases the risk of autism. Our goal in this research is to provide as much information as possible in this context. Estimating the penetrance of the CNV for autism is a key step in that process. Penetrance of BP1-BP2 CNVs is likely to be low because the vast majority of BP1-BP2 CNVs identified in clinical cohorts are inherited from healthy parents, and BP1-BP2 $\mathrm{CNVs}$ have been identified in control subjects. However, penetrance estimates vary widely across studies for the deletion (from 0.10 to 0.83) [Cooper et al., 2011; Rosenfeld, Coe, Eichler, Cuckle, \& Shaffer, 2013] and are lacking for the duplication. Moreover, the contribution of BP1-BP2 CNVs to ASD risk, as opposed to developmental disorders more broadly, has never been examined separately.
Characterization of parent and sibling carriers can also inform our understanding of the nature of risk and has proven fruitful in several instances [Girirajan \& Eichler, 2010; Zufferey et al., 2012]. Given the great phenotypic heterogeneity of ASD, which is thought to reflect the extensive complexity of the architecture of genetic risk for autism, studying subtle autistic symptoms in relatives can disentangle the effect of variants on phenotype. Furthermore, it is important to examine the relationship of $\mathrm{CNV}$ and phenotype in probands, such as sex-specific expression and the effect of parent of origin. To gain insight into the pathogenicity of the BP1-BP2 CNVs, we study a well-characterized ASD family sample ( $\mathrm{n}=2525$ families) from the Simons Foundation Autism Research Initiative (SFARI). To our knowledge, this is the first attempt to examine the pathogenicity of the BP1-BP2 CNVs specifically for ASD.

\section{Methods \\ ASD Families}

Included were a total of 2525 ASD families with both phenotype and genotype data available. These consist of 2482 families from the Simons Simplex Collection (SSC) (version 14.1), 31 families from the Simons Ancillary Collection (SAC), and 12 monozygotic twin families from the Simons Twin Collection (STC). The main properties of the families in the SSC have been described by Fischbach and Lord [Fischbach \& Lord, 2010]. Additional information on the SAC and STC families is included in the Supporting Information. The ASD sample consists of 2525 fathers, 2525 mothers, 2036 designated siblings (s1), and 2525 designated probands (p1). All individuals in this sample were older than 4 years. Further information regarding inclusion/exclusion criteria for SSC, SAC, and STC, as well as the complete list of instruments used to assess the phenotype of the families, are available on the SFARI website (https://sfari.org/).

\section{Phenotype Measures}

For parents, the average score of the Broad Autism Phenotype Questionnaire (BAPQ) [Hurley, Losh, Parlier, Reznick, $\&$ Piven, 2007] and the total raw score of the Social Responsiveness Scale (SRS) [Constantino \& Gruber, 2005], adult version (SRS-A) were included in the analysis. For siblings, the total T score of SRS-Parent version (SRS-P) was analyzed. The SRS-P score was not analyzed in the probands because the distribution of the scores showed a major ceiling effect in probands. Composite score of the Vineland Adaptive Behavior Scale-2nd edition (VABS-II) [Sparrow, Cicchetti, \& Bella, 2005] was analyzed in both probands and siblings, separately. For probands only, the effects of the CNVs on Calibrated Severity Scales (CSS) 
[Gotham, Pickles, \& Lord, 2009; Hus, Gotham, \& Lord, 2012] of Social affect (SA-CSS) and restricted repetitive behavior (RRB-CSS) from the Autism Diagnostic Observation Schedule-WPS edition [Lord, Rutter, DiLavore, \& Risi, 2006], and intellectual quotient (IQ) consisting of full scale IQ, verbal IQ (VIQ), and nonverbal IQ, were modeled. IQ scores were derived from one or more of the following instruments: Differential Ability Scales, 2nd edition [Elliott, 2007], Wechsler Intelligence Scale for Children, 4th edition [Wechsler, 2004], Mullen Scales of Early Learning [Mullen, 1995], or the Raven's standard progressive matrices [Raven, 1981]. When children had the Raven's, VIQ was estimated from the Peabody Picture Vocabulary Test-4th edition [Dunn \& Dunn, 2007].

\section{Microarray Analyses}

The ASD families were genotyped on the Illumina Infinium ${ }^{\circledR} 1 \mathrm{Mv} 1$ (338 families), Infinium ${ }^{\circledR} 1 \mathrm{Mv} 3$ Duo (1191 families), or the HumanOmni2.5-8 (996 families) microarrays (Illumina Inc, San Diego, CA, USA). All three microarray types had over 180 probes within the BP1-BP2 region. CNV prediction was performed by PennCNV (PN) [Wang et al., 2007], QuantiSNP (QT) [Colella et al., 2007], and Gnosis [Sanders et al., 2011], using CNVision [Sanders et al., 2011]. Detailed CNV detection protocol was described in [Sanders et al., 2011]. Sample identity within the family, including correct assignment of paternity and maternity, was confirmed using genetically inferred identity by descent for all study participants, including the de novo deletion of BP1-BP2 in a proband.

\section{Statistical Analysis}

The distributions of SRS scores (SRS-A and SRS-P) in unaffected relatives were not symmetric (Fig. S1). Because extreme observations (out in the tail) can act as "influence points" for regression effects, we analyzed all data using the square root of SRS scores. The effects of BP1-BP2 CNVs in parents on ASD-related phenotypes were examined using generalized estimating equations (GEE) to take into account the correlation between the mother and father SRS scores and the mother and father BAPQ scores. Linear regression was used to explore the results obtained with GEE further by analyzing the mothers and fathers separately. All analyses were done using R (http://cran.rproject.org). GEE was implemented using the gee package, with the identity variable being family; the correlation among family members was defined as fixed for the correlation between parents' scores ( 0.34 for SRS and 0.11 for BAPQ).

All the models initially included sex and age as covariates, as well as sex $\times \mathrm{CNV}$ interaction. Covariates were dropped when they were nonsignificant predictors, thereby identifying the most parsimonious model. For modeling the relationship between BP1-BP2 CNV and phenotype, we made the a priori assumption that both the deletion and duplication had a similar effect on phenotype: the relatively small number of both deletions and duplications would not permit distinction of subtle differences, even if they exist.

\section{Transmission Disequilibrium Test and Penetrance Calculations}

The transmission equilibrium of inherited CNVs in our ASD family sample was examined to further evaluate pathogenicity of the BP1-BP2 CNVs, using chi statistics. Penetrance estimate was calculated as a conditional probability: $\quad \mathbf{P}(\mathbf{D} \mid \mathbf{G})=\mathbf{P}(\mathbf{G} \mid \mathbf{D}) \times \mathbf{P}(\mathbf{D}) /[\mathbf{P}(\mathbf{G} \mid \mathbf{D}) \times \mathbf{P}(\mathbf{D})$ $+\mathbf{P}(\mathbf{G} \mid \sim \mathbf{D}) \times \mathbf{( 1 - P ( D ) )}$ ] [Vassos et al., 2010], in which $\mathbf{P}$ is probability, D means the subject is diagnosed with ASD, -D means the subject is unaffected, G encodes carrier status for BP1-BP2 CNV, and $\mathbf{P ( D | G )}$ is read as the probability of being diagnosed with ASD given the subject carries a BP1-BP2 CNV. The subtlety here is that this cohort was ascertained for affected probands, and thus to solve for the penetrance $\mathbf{P}(\mathbf{D} \mid \mathbf{G})$ we must reverse the conditional (e.g., $[\mathbf{P}(\mathbf{G} \mid \mathbf{D})$ ) and use an estimate of the prevalence of ASD, $\mathbf{P ( D )}$, which we take to be equal to 0.01. The penetrance estimate was performed separately for deletion and duplication because published results from other cohorts suggest they differ somewhat in their penetrance for other developmental and neuropsychiatric outcomes [Cooper et al., 2011; Kirov et al., 2013]. We also estimated the penetrance for the deletion and duplication combined.

\section{Results}

Estimate of Frequency and Penetrance of BP1-BP2 CNVS

Eighty-nine subjects in 47 families carried a CNV involving the BP1-BP2 region of chromosome 15. Among those, four families were excluded from all analyses due to the presence of a larger 15q11.2-13 CNV involving the PWS/AS region (Table S1); all four were de novo CNVs in probands. One father-proband pair carried a BP1-BP2 duplication involving only two genes (TUBGCP5 and CYFIP1); this pair was included in the analyses. In the remaining 85 subjects in 43 families, the BP1-BP2 deletion was observed in 6/2525 fathers, 8/2525 mothers, 5/2036 siblings, and 8/2521 probands, whereas BP1-BP2 duplication was observed in 16/2525 fathers, $13 / 2525$ mothers, 9/2036 siblings, and 20/2521 probands. So the rates of deletion (duplication) were $0.28 \%(0.57 \%)$ in parents, $0.25 \%(0.44 \%)$ in unaffected siblings (s1), and $0.32 \%(0.79 \%)$ in probands, respectively (Table 1$)$.

Only one CNV, a deletion, was de novo and it was transmitted by the father. Neither the frequency of the 
deletion nor the frequency of the duplication differed significantly between ASD probands and siblings ( $P=0.86$ and $P=0.19$, respectively). The frequency of the deletion was comparable to published rates in controls (0.18-0.38\%) [Cooper et al., 2011; Rosenfeld et al., 2013; Stefansson et al., 2008] and somewhat lower than in individuals with schizophrenia (0.59\% [Kirov et al., 2013] to $0.62 \%$ [Vassos et al., 2010]), or in subjects with developmental delay $(0.60 \%$ [Cooper et al., 2011] to $0.81 \%$ [Rosenfeld et al., 2013]). The frequency of the duplication in ASD probands was higher than the previously published rates in both cases (0.41\% [Cooper et al., 2011]) and controls (0.34\% [Stefansson et al., 2008] to $0.43 \%$ [Cooper et al., 2011]). No parent-of-origin-specific distortion of parental transmission was observed in this sample (Table S2).

The estimate of penetrance was 0.013 for the deletion and 0.018 for the duplication (0.016 for either), given a prevalence of 0.01 for ASD [Centers for Disease Control, CDC, 2006, 2012], which means that the presence of the BP1-BP2 deletion/duplication would increase the risk of having autism by about three in a thousand for the deletion and eight in a thousand for the duplication. However, the confidence interval (CI) of the odds ratio (OR) is quite large (deletion: $\mathrm{OR}=1.3 \mathrm{CI} 95 \%=[0.42-$ 3.96]; duplication OR $=1.8$, CI 95\% $=[0.82-3.97]$ ).

\section{Transmission Analysis of BP1-BP2 CNVs}

There was no preferential transmission of the deletion, either in probands or siblings in this sample, whereas the duplication was preferentially transmitted from parents

Table 1. BP1-BP2 CNV Frequency in 2525 ASD Families

\begin{tabular}{lrrrr}
\hline 15q11.2 CNV & Father & Mother & \multicolumn{1}{c}{$s 1$} & \multicolumn{1}{c}{ 1 1} \\
\hline BP1-BP2 deletion & 6 & 8 & 5 & 8 \\
BP1-BP2 duplication & 16 & 13 & 9 & 20 \\
No CNV & 2503 & 2504 & 2022 & 2493 \\
CNV overlapping the PWS/AS CR & 0 & 0 & 0 & 4 \\
Sum & 2525 & 2525 & 2036 & 2525 \\
\hline
\end{tabular}

ASD, autism spectrum disorder; CNV, copy number variant; PWS/AS CR, Prader-Willi/Angelman Syndrome Critical Region (BP2-BP3); s1, designated sibling; $\mathrm{p} 1$, designated proband. to probands (20T vs. 9NT, $\mathrm{X}^{2}=4.2$, one-tailed $P=0.041$ ) but not to unaffected siblings (Table S3 and Fig. S2). Interestingly, preferential transmission of duplication was most evident in trio families (10T vs. 0NT) vs. quartet families (10T vs. 9NT). We explored further the transmission results for "stoppage effect (stop having children after the proband)" in relation to birth orders of the probands. We did not observe any meaningful pattern between trio and quartet families. Transmission to siblings and probands appears similar between fathers and mothers.

\section{Effects of BP1-BP2 CNVs on Autism-Related Symptoms in Relatives}

In parents, the model including both sex and sex $\times \mathrm{CNV}$ interaction revealed nominally significant $\mathrm{CNV}$ effect on SRS score in parents (Table 2). When the model was simplified, however, by dropping age (nonsignificant effect) and sex $\times \mathrm{CNV}$ interaction (close to significance), the CNV effect on SRS score became not significant. Because these results suggest that there may be a modest difference between mothers and fathers in the effect of the $\mathrm{CNV}$, data from mothers and data from fathers were analyzed separately. The results (Table S4) were consistent with a greater effect of the CNV on SRS scores in fathers than mothers. GEE analysis for BAPQ score was not significant with or without sex and age included in the model (Table 2).

In siblings (Table S4), a CNV effect on SRS score was not observed either overall or in male siblings only (estimate $-0.08946, P$ value 0.67$)$; however, there was a significant effect of "parent-of-origin" (PoO) on SRS score (Table S4), specifically CNVs from mothers had a greater impact than those from fathers. Thus, there appeared to be modest heterogeneity by PoO, but it is important to also note the small sample size of 13 siblings (one did not have SRS data). Last, while the analysis of the effect of the CNV on composite score of VABS in siblings did not reveal a significant $\mathrm{CNV}$ effect, the results of the model including PoO, sex, and an interaction between PoO and sex suggested a trend toward a greater effect in males who received the $\mathrm{CNV}$ from their mother.

Table 2. Effect of CNV on SRS (Square Root) and BAPQ Scores in Parents Using Generalized Estimating Equations

\begin{tabular}{|c|c|c|c|c|c|c|c|}
\hline Clinical Measure & Predictors & Estimate & Naïve SE & Naïve z & Robust SE & Robust z & $P$ value \\
\hline \multirow[t]{3}{*}{ SRS } & CNV & 1.04661980 & 0.41466441 & 2.524016 & 0.53327662 & 1.962621 & 0.0497 \\
\hline & Sex & 0.05556904 & 0.05480882 & 1.013870 & 0.05473152 & 1.015302 & 0.31 \\
\hline & Sex $\times$ CNV & -1.09070467 & 0.59330134 & -1.838365 & 0.65829129 & -1.656872 & 0.0975 \\
\hline \multirow[t]{2}{*}{ BAPQ } & CNV & 0.1122471 & 0.09024223 & 1.243842 & 0.09343174 & 1.20138 & 0.23 \\
\hline & sex & -0.2915663 & 0.01665541 & -17.505799 & 0.01665045 & -17.51102 & $1.18 \times 10^{-68}$ \\
\hline
\end{tabular}

SRS, Social Responsiveness Scale; BAPQ, Broad Autism Phenotype Questionnaire; sex $\times$ CNV, interaction between sex and CNV; CNV, copy number variant. 


\section{The Effects of BP1-BP2 CNVs in Probands}

Before analyses, two probands were excluded from the phenotype/genotype analyses because they carried a large de novo $\mathrm{CNV}$ (3.5 Mb and $5.4 \mathrm{Mb}$ respectively) in another chromosome (Table S1). Performing the same stepwise analyses described earlier, neither the analysis of the effect of $\mathrm{CNV}$ nor the analysis of $\mathrm{PoO}$ supported a large effect of the CNV on any of the analyzed phenotypes (Table S5), and there was no consistency between the results of the different models.

\section{Discussion}

ASD is a group of heterogeneous neurodevelopmental disorders causing significant social, communication, and behavioral deficits and challenges [APA, 2000]. While genome-wide genotyping and sequencing are beginning to elucidate the complex architecture of autism risk, the contribution of inherited rare variation to genetic risk for autism remains elusive [Bucan et al., 2009; Girirajan, Johnson et al., 2013; Krumm et al., 2013; Morrow et al., 2008; Pinto et al., 2010; Sanders et al., 2011, 2012]. Although this contribution is likely to be low in terms of proportion of phenotype explained in a sample (i.e., heritability related to rare inherited variation), it is an important issue at the individual level because of the consequences for genetic counseling. Moreover, the discovery of some inherited CNVs occurring at loci affected by de novo CNVs shows that some rare inherited variation carries risk for ASD. To gain further insight into the pathogenicity of the BP1-BP2 CNVs, which have been proposed as risk factors for ASD, we have estimated the penetrance of the CNV and effects on ASD phenotype in a well-defined ASD family sample.

Intriguingly, the results obtained in this sample are different than the results from previous studies on broader neurodevelopmental phenotypes [Cooper et al., 2011]. Indeed, there was no evidence of association between the deletion and ASD in this sample, whereas a significant over-transmission of the duplication to probands was observed. Moreover, the estimated penetrance was lower for the deletion than for the duplication (0.013 vs. 0.018). This could be the consequence of an ascertainment bias. For this sample, parents were selected so that they did not present with even a mild autism phenotype, and they would thus be different from the unscreened parental population. A relevant observation is that the frequency of BP1-BP2 deletion in both unaffected relatives and probands was similar to published rates in controls [Cooper et al., 2011; Rosenfeld et al., 2013; Stefansson et al., 2008]. While it is possible that differences in CNV detection confound interpretation of these data, another plausible explanation for these differences is that the recruitment modalities of the SFARI lead to the selection of families less likely to carry rare, inherited ASD risk variation, such as BP1-BP2 CNVs. Certainly, this was the purpose of the strict family assessment of the SFARI, and its impact has been documented for common variants affecting risk for ASD [Klei et al., 2012]. While in this study the penetrance of the duplication was estimated to be slightly higher than the penetrance of the deletion, this could also be a result of ascertainment bias: families with the duplication being less likely to have been excluded because the duplication has smaller phenotypic effect, while the smaller sample size of parents carrying deletions translates to greater variance in transmission than that for duplications. The sole BP1-BP2 de novo event, a deletion, lends some credence to this possibility. Thus, it is possible, given these results, that the impact of BP1-BP2 on risk is somewhat underestimated by our study. Nonetheless, there can be no doubt that its impact is modest and its penetrance is low.

All other results are consistent with a very modest effect. Interestingly, the results of the regression analysis of the effect of the CNVs on SRS scores suggested that males might be more sensitive to the effect of the CNV, with a greater effect of CNVs in fathers than in mothers. Still, there is no effect of presence of the CNV on the score for the BAPQ for any model. It is tempting to say that these results show that the impact of the CNV is principally on sociability unrelated to broader autism phenotypes, but that interpretation must be tempered by the ascertainment used to recruit parents for the study, namely that they show few if any traits of the broader autism phenotype. This confounding limits our ability to interpret negative findings, but bolsters our confidence in positive results, which move contrary to expectations based on the ascertainment of parents for this study.

When we evaluated the effects of the BP1-BP2 CNVs on the severity of autism phenotype in probands, we did not observe any effect of the CNV in a positive or negative direction. This is consistent with the low penetrance and low impact on risk, suggesting this CNV and other risk factors, perhaps many, are required for a carrier to be diagnosed with ASD. The results yield a glimpse of the great challenge of identifying rare inherited variants that increase risk for autism.

To our knowledge, this is the first systematic study to investigate the effect of the BP1-BP2 CNVs on ASD risk. The results suggest that BP1-BP2 CNVs confer very little additional risk for ASD. One intriguing observation is that the effect of maternally inherited CNVs effect could be slightly higher than attributable to paternally inherited CNVs. However, these results should be considered as exploratory, and larger samples will be required to understand if this effect is confirmed or simply a stochastic effect due to small sample size and multiple testing. The main limitation of this study is the relatively small sample size of carriers, consistent with the rarity of the BP1-BP2 CNVs in the population in general. To replicate 
our results on penetrance with good power $(80 \%$, with $\alpha=0.05)$, for example, a very large sample size will be required (20027 probands and controls for the deletion, 3466 probands controls for the duplication). However, a strength of this study is that the family-based analyses avoid population structure bias, which is likely to be important in studies of rare variants [Liu, Fast, Zawistowski, \& Tintle, 2013]. In any case, the results of this study strongly suggest that the effect on ASD risk is small. Following the current American College of Medical Genetics guidelines for interpretation of postnatal CNV [Kearney, Thorland, Brown, Quintero-Rivera, \& South, 2011], these CNVs remain classified as variants of unknown significance (VOUS).

\section{Acknowledgments}

We are grateful to all of the families at the participating Simons Simplex Collection (SSC) sites, as well as the principal investigators (A. Beaudet, R. Bernier, J. Constantino, E. Cook, E. Fombonne, D. Geschwind, R. Goin-Kochel, E. Hanson, D. Grice, A. Klin, D. Ledbetter, C. Lord, C. Martin, D. Martin, R. Maxim, J. Miles, O. Ousley, K. Pelphrey, B. Peterson, J. Piggot, C. Saulnier, M. State, W. Stone, J. Sutcliffe, C. Walsh, Z. Warren, E. Wijsman). We appreciate obtaining access to phenotypic data on the Simons Foundation Autism Research Initiative (SFARI) Base. Approved researchers can obtain the SSC population dataset described in this study (http:// sfari.org/resources/sfari-base) by applying at https:// base.sfari.org. This work was supported by a grant from the Simons Foundation (SFARI 124827 to the investigators of the SSC Genetic Consortium), NIH K23MH082883 (SJK), NIH R01HD065272 (CL), NIH R01MH089390 (CL), CIHR (Doctoral Research Award to AJW), HHMI (International Student Research Fellowship to SJS), and Fondamental Foundation (PC).

\section{Conflict of Interest}

Dr. Cathy Lord receives royalties from Western Psychological Service for the sale of diagnostic instruments which she has coauthored. She donates all royalties from her projects and clinics to not-for-profit autism charities, specifically Have Dreams which is located in Chicago, Illinois. Have Dreams offers wraparound services for children and adults with autism. Dr. Arthur L. Beaudet is a Professor in the Department of Molecular and Human Genetics at Baylor College of Medicine. He plays a leading role in the operation of genetic diagnostic laboratories based in the department which derives revenue from this activity. The laboratories offer chromosomal microarray analysis, exome sequencing, and many other tests. Dr. Daniel Geschwind is a consultant for synapDx. Dr. Timothy Yu has held stock in and serves as a consultant for Claritas
Genomics, a DNA diagnostics company. All other authors reported no biomedical financial interests or potential conflicts of interest.

\section{References}

Abdelmoity, A.T., LePichon, J.B., Nyp, S.S., Soden, S.E., Daniel, C.A., \& Yu, S. (2012). 15q11.2 proximal imbalances associated with a diverse array of neuropsychiatric disorders and milddysmorphic features. Journal of Developmental and Behavioral Pediatrics, 33, 570-576.

APA. (2000). Diagnostic and statistical manual of mental disorders, text revision (DSM-IV-TR) (4th ed.). Washington, DC: American Psychiatric Publishing.

Bucan, M., Abrahams, B.S., Wang, K., Glessner, J.T., Herman, E.I., et al. (2009). Genome-wide analyses of exonic copy number variants in a family-based study point to novel autism susceptibility genes. PLoS Genetics, 5, e1000536.

Burnside, R.D., Pasion, R., Mikhail, F.M., Carroll, A.J., Robin, N.H., et al. (2011). Microdeletion/microduplication of proximal 15q11.2 between BP1 and BP2: A susceptibility region for neurological dysfunction including developmental and language delay. Human Genetics, 130, 517528.

Butler, M.G., Bittel, D.C., Kibiryeva, N., Talebizadeh, Z., Thompson, T. (2004). Behavioral differences among subjects with Prader-Willi syndrome and type I or type II deletion and maternal disomy. Pediatrics, 113, 565-573.

CDC. (2006). Mental health in the United States: Parental report of diagnosed autism in children aged 4-17 years-United States, 2003-2004. Morbidity and Mortality Weekly Report, $55,481-486$.

CDC. (2012). Prevalence of autism spectrum disorders-Autism and developmental disabilities monitoring network, 14 sites, United States, 2008. Morbidity and Mortality Weekly Report: Surveillance Summaries, 61, 1-19.

Christian, S.L., Fantes, J.A., Mewborn, S.K., Huang, B., Ledbetter, D.H. (1999). Large genomic duplicons map to sites of instability in the Prader-Willi/Angelman syndrome chromosome region (15q11-q13). Human Molecular Genetics, 8, 10251037.

Colella, S., Yau, C., Taylor, J.M., Mirza, G., Butler, H., et al. (2007). QuantiSNP: An objective Bayes Hidden-Markov model to detect and accurately map copy number variation using SNP genotyping data. Nucleic Acids Research, 35, 20132025.

Constantino, J.N., \& Gruber, C.P. (2005). Social Responsiveness Scale (SRS). Los Angeles, CA: Western Psychological Services.

Cook, E.H., Jr., Lindgren, V., Leventhal, B.L., Courchesne, R., Lincoln, A., et al. (1997). Autism or atypical autism in maternally but not paternally derived proximal $15 \mathrm{q}$ duplication. American Journal of Human Genetics, 60, 928-934.

Cooper, G.M., Coe, B.P., Girirajan, S., Rosenfeld, J.A., Vu, T.H., et al. (2011). A copy number variation morbidity map of developmental delay. Nature Genetics, 43, 838-846.

de Kovel, C.G., Trucks, H., Helbig, I., Mefford, H.C., Baker, C., et al. (2010). Recurrent microdeletions at 15q11.2 and 16p13.11 predispose to idiopathic generalized epilepsies. Brain: A Journal of Neurology, 133, 23-32. 
Devlin, B., \& Scherer, S.W. (2012). Genetic architecture in autism spectrum disorder. Current Opinion in Genetics and Development, 22, 229-237.

Doornbos, M., Sikkema-Raddatz, B., Ruijvenkamp, C.A., Dijkhuizen, T., Bijlsma, E.K., et al. (2009). Nine patients with a microdeletion 15q11.2 between breakpoints 1 and 2 of the Prader-Willi critical region, possibly associated with behavioural disturbances. European Journal of Medical Genetics, 52, 108-115.

Dunn, L.M., \& Dunn, D.M. (2007). Peabody picture vocabulary test, fourth edition (PPVT-IV). A measure of receptive vocabulary for Standard American English, San Antonio, TX: Pearson.

Elliott, C.D. (2007). Differential Ability Scale (2nd ed.). San Antonio, TX: Psychological Corporation.

Fischbach, G.D., \& Lord, C. (2010). The Simons Simplex Collection: A resource for identification of autism genetic risk factors. Neuron, 68, 192-195.

Girirajan, S., Dennis, M.Y., Baker, C., Malig, M., Coe, B.P., et al. (2013). Refinement and discovery of new hotspots of copynumber variation associated with autism spectrum disorder. American Journal of Human Genetics, 92, 221-237.

Girirajan, S., \& Eichler, E.E. (2010). Phenotypic variability and genetic susceptibility to genomic disorders. Human Molecular Genetics. 19, R176-R187.

Girirajan, S., Johnson, R.L., Tassone, F., Balciuniene, J., Katiyar, N., et al. (2013). Global increases in both common and rare copy number load associated with autism. Human Molecular Genetics, 22, 2870-2880.

Gotham, K., Pickles, A., \& Lord, C. (2009). Standardizing ADOS scores for a measure of severity in autism spectrum disorders. Journal of Autism and Developmental Disorders, 39, 693-705.

Hartley, S.L., Maclean, W.E., Jr., Butler, M.G., Zarcone, J., \& Thompson, T. (2005). Maladaptive behaviors and risk factors among the genetic subtypes of Prader-Willi syndrome. American Journal of Medical Genetics. Part A, 136, 140-145.

Hurley, R.S., Losh, M., Parlier, M., Reznick, J.S., \& Piven, J. (2007). The Broad Autism Phenotype Questionnaire. Journal of Autism and Developmental Disorders, 37, 1679-1690

Hus, V., Gotham, K., \& Lord, C. (2012). Standardizing ADOS domain scores: Separating severity of social affect and restricted and repetitive behaviors. Journal of Autism and Developmental Disorders, 2012 Nov 11.

Kearney, H.M., Thorland, E.C., Brown, K.K., Quintero-Rivera, F., $\&$ South, S.T. (2011). American College of Medical Genetics standards and guidelines for interpretation and reporting of postnatal constitutional copy number variants. Genetics in Medicine, 13, 680-685.

Kirov, G., Rees, E., Walters, J.T., Escott-Price, V., Georgieva, L., et al. (2013). The penetrance of copy number variations for schizophrenia and developmental delay. Biological Psychiatry, 75, 378-385.

Klei, L., Sanders, S.J., Murtha, M.T., Hus, V., Lowe, J.K., et al. (2012). Common genetic variants, acting additively, are a major source of risk for autism. Molecular Autism, 3, 9.

Krumm, N., O'Roak, B.J., Karakoc, E., Mohajeri, K., Nelson, B., et al. (2013). Transmission disequilibrium of small CNVs in simplex autism. American Journal of Human Genetics, 93, 595-606.
Liu, K., Fast, S., Zawistowski, M., \& Tintle, N.L. (2013). A geometric framework for evaluating rare variant tests of association. Genetic Epidemiology, 37, 345-357.

Lord, C., Rutter, M., DiLavore, P.C., \& Risi, S. (2006). Autism Diagnostic Observation Schedule WPS edition manual. Los Angeles, CA: Western Psychological Services.

Moreno-De-Luca, D., Sanders, S.J., Willsey, A.J., Mulle, J.G., Lowe, J.K., et al. (2012). Using large clinical data sets to infer pathogenicity for rare copy number variants in autism cohorts. Molecular Psychiatry, 18, 1090-1095.

Morrow, E.M., Yoo, S.Y., Flavell, S.W., Kim, T.K., Lin, Y., et al. (2008). Identifying autism loci and genes by tracing recent shared ancestry. Science, 321, 218-223.

Mullen, E.M. (1995). The Mullen Scales of Early Learning. Circle Pines, MN: American Guidance Service Inc.

Murthy, S.K., Nygren, A.O., El Shakankiry, H.M., Schouten, J.P., Al Khayat, A.I., et al. (2007). Detection of a novel familial deletion of four genes between BP1 and BP2 of the PraderWilli/Angelman syndrome critical region by oligo-array CGH in a child with neurological disorder and speech impairment. Cytogenetic and Genome Research, 116, 135-140.

Pinto, D., Pagnamenta, A.T., Klei, L., Anney, R., Merico, D., et al. (2010). Functional impact of global rare copy number variation in autism spectrum disorders. Nature, 466, 368372.

Raven, J. (1981). Manual for Raven's progressive matrices and vocabulary scales. Research Supplement No.1: The 1979 British Standardisation of the Standard Progressive Matrices and Mill Hill Vocabulary Scales, Together With Comparative Data From Earlier Studies in the UK, US, Canada, Germany and Ireland. San Antonio, TX, Harcourt Assessment.

Rosenfeld, J.A., Coe, B.P., Eichler, E.E., Cuckle, H., \& Shaffer, L.G. (2013). Estimates of penetrance for recurrent pathogenic copy-number variations. Genetics in Medicine, 15, 478481.

Sahoo, T., Bacino, C.A., German, J.R., Shaw, C.A., Bird, L.M., et al. (2007). Identification of novel deletions of 15q11q13 in Angelman syndrome by array-CGH: Molecular characterization and genotype-phenotype correlations. European Journal of Human Genetics, 15, 943-949.

Sanders, S.J., Ercan-Sencicek, A.G., Hus, V., Luo, R., Murtha, M.T., et al. (2011). Multiple recurrent de novo CNVs, including duplications of the 7q11.23 Williams syndrome region, are strongly associated with autism. Neuron, 70, 863-885.

Sanders, S.J., Murtha, M.T., Gupta, A.R., Murdoch, J.D., Raubeson, M.J., et al. (2012). De novo mutations revealed by whole-exome sequencing are strongly associated with autism. Nature, 485, 237-241.

Sempere Perez, A., Manchon Trives, I., Palazon Azorin, I., Alcaraz Mas, L., Perez Lledo, E., \& Galan Sanchez, F. (2011). 15q11.2 (BP1-BP2) microdeletion, a new syndrome with variable expressivity. Anales de Pediatria (Barcelona, Spain: 2003), 75, 58-62.

Shen, Y., Dies, K.A., Holm, I.A., Bridgemohan, C., Sobeih, M.M., et al. (2010). Clinical genetic testing for patients with autism spectrum disorders. Pediatrics, 125, e727-e735.

Sparrow, S.S., Cicchetti, D.V., \& Bella, D.A. (2005). Vineland Adaptive Behavior Scales (2nd ed.). Minneapolis, MN: NCS Pearson, Inc. 
Stefansson, H., Rujescu, D., Cichon, S., Pietilainen, O.P., Ingason, A., et al. (2008). Large recurrent microdeletions associated with schizophrenia. Nature, 455, 232-236.

van der Zwaag, B., Staal, W.G., Hochstenbach, R., Poot, M., Spierenburg, H.A., et al. (2010). A co-segregating microduplication of chromosome $15 \mathrm{q} 11.2$ pinpoints two risk genes for autism spectrum disorder. American Journal of Medical Genetics. Part B, Neuropsychiatric Genetics, 153B, 960-966.

von der Lippe, C., Rustad, C., Heimdal, K., \& Rodningen, O.K. (2011). 15q11.2 microdeletion-Seven new patients with delayed development and/or behavioural problems. European Journal of Medical Genetics, 54, 357-360.

Vassos, E., Collier, D.A., Holden, S., Patch, C., Rujescu, D., et al. (2010). Penetrance for copy number variants associated with schizophrenia. Human Molecular Genetics, 19, 3477-3481.

Wang, K., Li, M., Hadley, D., Liu, R., Glessner, J., et al. (2007). PennCNV: An integrated hidden Markov model designed for high-resolution copy number variation detection in wholegenome SNP genotyping data. Genome Research, 17, 16651674.

Wechsler, D. (2004). The Wechsler Intelligence Scale for Children (4th ed.). London, UK: Pearson Assessment.

Weiss, L.A., Shen, Y., Korn, J.M., Arking, D.E., Miller, D.T., et al. (2008). Association between microdeletion and microduplication at 16p11.2 and autism. The New England Journal of Medicine, 358, 667-675.

Zufferey, F., Sherr, E.H., Beckmann, N.D., Hanson, E., Maillard, A.M., et al. (2012). A $600 \mathrm{~kb}$ deletion syndrome at $16 \mathrm{p} 11.2$ leads to energy imbalance and neuropsychiatric disorders. Journal of Medical Genetics, 49, 660-668.

\section{Supporting Information}

Additional Supporting Information may be found in the online version of this article at the publisher's web-site:

Appendix S1. Description of SAC and STC families included in the study.

Figure S1. Distribution of SRS scores. Fathers SRS scores: untransformed (left) and square root transformed (right).

Figure S2. Frequencies of transmitted vs. not transmitted CNVs in probands and siblings.

Table S1. List of probands (p1) excluded from the analyses.

Table S2. Inheritance of BP1-BP2 CNV in siblings and probands of ASD families.

Table S3. Transmission of BP1-BP2 CNVs.

Table S4. Effect of CNV on phenotype in parents and siblings estimated from multiple linear regression analysis.

Table S5. Effect of CNV on phenotype in probands estimated from multiple linear regression analysis. 